\title{
Software-Programmed Optical Networking with Integrated NFV Service Provisioning
}

Mehmeri, Victor; Wang, Xi; Basu, Shrutarshi; Zhang, Qiong; Palacharla, Paparao; Ikeuchi, Tadashi; Tafur Monroy, Idelfonso ; Vegas Olmos, Juan José; Foster, Nate

Published in:

Optical Fiber Communication Conference 2017

Link to article, DOI:

10.1364/OFC.2017.Tu3L.12

Publication date:

2017

Document Version

Peer reviewed version

Link back to DTU Orbit

Citation (APA):

Mehmeri, V., Wang, X., Basu, S., Zhang, Q., Palacharla, P., Ikeuchi, T., Tafur Monroy, I., Vegas Olmos, J. J., \& Foster, N. (2017). Software-Programmed Optical Networking with Integrated NFV Service Provisioning. In Optical Fiber Communication Conference 2017 Optical Society of America.

https://doi.org/10.1364/OFC.2017.Tu3L.12

\section{General rights}

Copyright and moral rights for the publications made accessible in the public portal are retained by the authors and/or other copyright owners and it is a condition of accessing publications that users recognise and abide by the legal requirements associated with these rights.

- Users may download and print one copy of any publication from the public portal for the purpose of private study or research.

- You may not further distribute the material or use it for any profit-making activity or commercial gain

- You may freely distribute the URL identifying the publication in the public portal 


\title{
Software-Programmed Optical Networking with Integrated NFV Service Provisioning
}

\author{
Victor Mehmeri ${ }^{1,2}$, Xi Wang ${ }^{1}$, Shrutarshi Basu ${ }^{3}$, Qiong Zhang ${ }^{1}$, Paparao Palacharla ${ }^{1}$, \\ Tadashi Ikeuchi $^{1}$, Idelfonso Tafur Monroy ${ }^{2}$, Juan Jose Vegas Olmos ${ }^{2}$, Nate Foster ${ }^{3}$ \\ ${ }^{1}$ Fujitsu Laboratories of America, Inc. 2801 Telecom Pkwy, Richardson, TX, USA, Email: vime@fotonik.dtu.dk \\ ${ }^{2}$ Technical University of Denmark, Department of Photonics Engineering, Lyngby 2800, Denmark \\ ${ }^{3}$ Cornell University, Department of Computer Science, Ithaca, NY, USA
}

\begin{abstract}
We showcase demonstrations of "program \& compile" styled optical networking as well as open platforms \& standards based NFV service provisioning using a proof-of-concept implementation of the Software-Programmed Networking Operating System (SPN OS).

OCIS codes: (060.4253) Networks; (060.4510) Optical Communications
\end{abstract}

\section{Overview}

This demonstration features "program \& compile" styled optical networking following the Software-Programmed Networking (SPN) paradigm [1]. SPN leverages network programming to represent a virtual network service as a Virtual Network Object (VNO), which enables the decoupling of distributed control of individual services from centralized management of network-wide resources. The virtual network service offered by a VNO is written in network programs using high level network programming language (e.g., NetKAT [2]), which can be compiled into device-level SDN commands (e.g., OpenFlow) upon service activation for automated configuration of the physical networks. Moreover, a VNO also provides built-in methods for various user-originated service operations (e.g., traffic rerouting, virtual topology reconfiguration, etc.), thus allowing flexible and distributed control of network services by individual users, as opposed to network-wide centralized control \& management in a typical SDN platform.

To allow for a comprehensive cross-layer network virtualization strategy that delivers flexibility at both packet and optical layers, while supporting dynamic Service Function Chaining (SFC) deployments to chain L4-L7 network functions over the network, we build an SPN Operating System (SPN OS) prototype to highlight the new capability of software-programmed optical networking with integrated NFV service provisioning. The overview of the SPN OS prototype is shown in Fig. 1. Using the recent enhancements of Open vSwitch (OVS) with support for Network Service Headers (NSH) [3] protocol, we showcase a VNO that encapsulates a service layer for end-to-end NFV SFC provisioning, a network layer comprising both packet and optical sublayers for cross-layer virtualization, and a built-in management suite for service operation. For network programing, we use NetKAT to program both packet and optical layers. For optical network configuration, we use LINC-OE [4] and OpenFlow 1.3 with optical extensions to emulate and control Reconfigurable Add-Drop Multiplexers (ROADM) nodes in a wide-area optical network. In addition, we use TOSCA templates to define SFCs which are dynamically rendered by a NFV manager (NFVM) based on OpenDaylight (ODL), which is also responsible for instantiating Virtual Network Functions (VNFs) on servers at the packet layer using lightweight Docker containers.
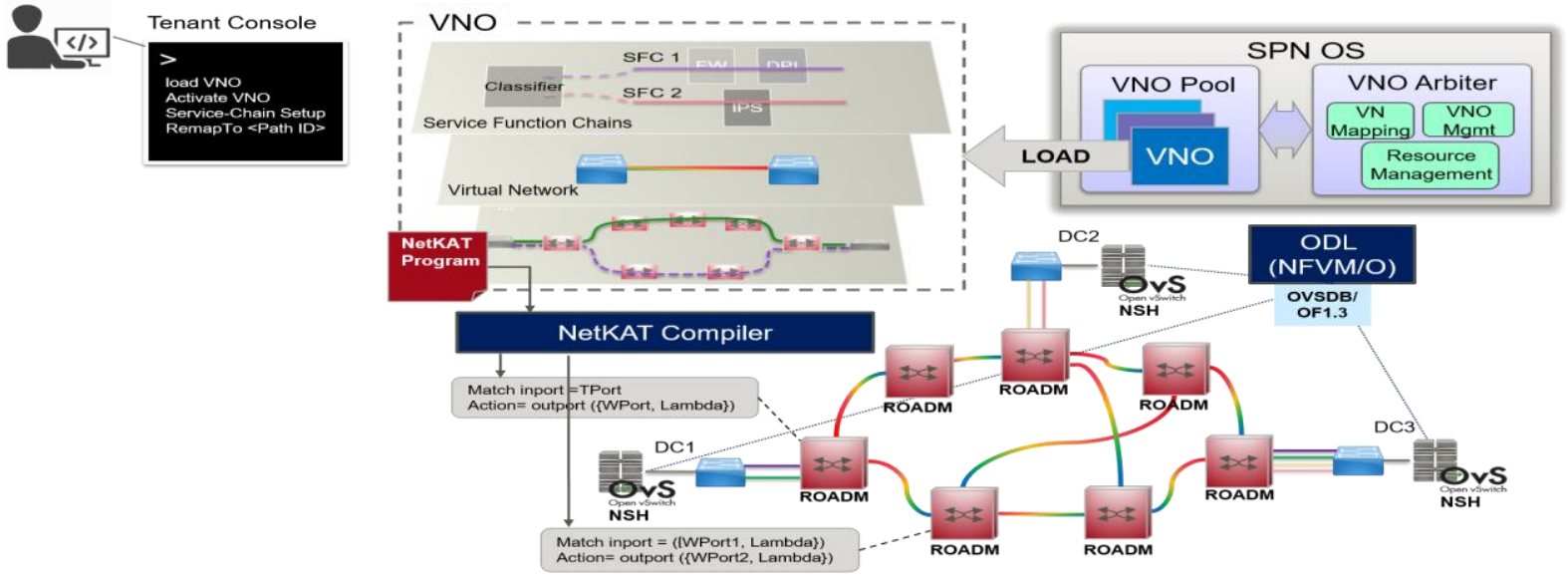

Fig. 1. SPN OS demo overview. VNO: Virtual Network Object; NFVM/O: Network Function Virtualization Manager/Orchestrator. 
We adopt NSH protocol for dynamic traffic steering using OVS as an SFC classifier, which injects traffic (as per template-defined Access Control List rules in our prototype) into a VxLAN tunnel associated with a particular chain based on NSH header fields. An overview and screenshots of an SFC created in our demo is shown in Fig. 2.

Steps to be demonstrated with our prototype system from a tenant console include: 1) VNO Instantiation, where a virtual network service and its management program are loaded by the Arbiter (in the SPN OS, the Arbiter is in charge of centralized resource management including computing the virtual-to-physical mapping and VNO creation), the physical network resources are allocated, and physical layer as well as virtual-to-physical mapping information are added to the VNO object; 2) VNO Activation, with which the VNO's programs get compiled by the NetKAT compiler, and OpenFlow rules are subsequently installed on the ROADM nodes, as well as the emulated packet switches (OVSs) to establish end-to-end network connectivity. 3) VNO Service Function Chain Setup, which configures the end-hosts to establish VxLAN-based tunnels for SFC connectivity. At this point VNFs are dynamically created and OpenFlow rules for SFC traffic steering are installed in the OVS data plane; 4) VNO Optical Remap, with which the optical layer can be remapped to a pre-programmed alternative optical path (e.g. an optical path with higher capacity that can be selected on-demand) through a re-compilation of the NetKAT program for the optical layer. Steps 2 through 4 are solely operated by the VNO without involvement of the Arbiter.

We also demonstrate traffic isolation between multi-tenant VNOs and the distributed control autonomy of SPN OS by performing optical layer control operations on an active VNO even after shutting down the Arbiter.
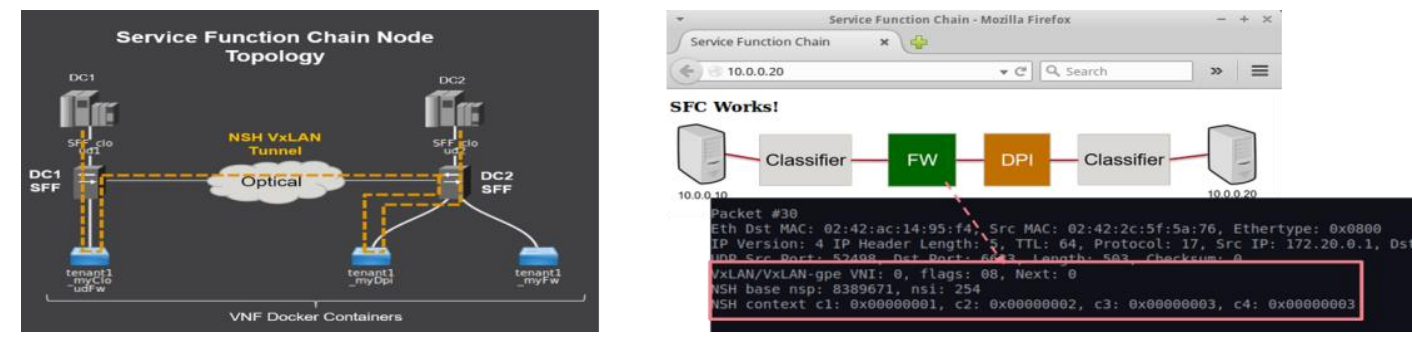

Fig. 2. Demonstration of dynamic SFC creation over a software-programmed optical network.

\section{Innovation Highlights}

SDN and NFV have dramatically increased the value creation of network services in datacenters, with more efficient resource utilization and management as well as the emergence of new business models out of multi-tenant based infrastructure virtualization. Meanwhile, many developments in metro and long-haul optical networks have been focused primarily on next-generation ROADM capabilities to achieve significant gains in capacity and flexibility. However, there is a pressing need for comprehensive network virtualization solutions incorporating packet as well as optical transport layer, allowing a cross-layered management approach and fine-grained control of the optical "pipes", in order to deliver full top-down flexibility to today's dynamic and software-defined network services.

In this proof-of-concept, we demonstrate how network programming can be leveraged to deliver flexible optical networking through the compilation of high-level policies into a set of "low-level" optical layer configurations (e.g. ROADM's OpenFlow tables). Use cases for such a system include: optical network as a service for dynamic interconnection of datacenters; fine-grained protection and restoration; scheduled remapping of optical path or ondemand selection of higher capacity optical path for bandwidth-on-demand offerings, etc.

\section{OFC Relevance}

As network services and applications migrate to cloud-based architectures where virtualization plays an essential role, it is important to incorporate optical networking into the end-to-end virtualization solutions. With the evolution and softwarization of flexible ROADMs and multi-rate, multi-modulation transponders, it is now imperative to support optical network virtualization using a comprehensive and expressive abstraction. To that end, this demonstration suggests one feasible solution, using the abstraction of a VNO to encapsulate layers of network services in a top-down manner, allowing fine-grained control over aspects of the underlying optical infrastructure. Based on such abstractions, we expect that seamless Optical Network as a Service (ONaaS) for supporting modern NFV applications can be offered with high level of flexibility, scalability, and reliability.

\section{References}

[1] X. Wang, C. Chen, P. Palacharla, M. Sekiya, S. Smolka, and N. Foster, "SPN OS: Managing Network Services with Virtual Network Objects," Proc. IEEE NFV-SDN 2015, pp. 151-157, Nov 18-21, San Francisco, CA.

[2] C. J. Anderson, N. Foster, A. Guha, J.-B. Jeannin, D. Kozen, C. Schlesinger, and D. Walker, "NetKAT: semantic foundations for networks," ACM SIGPLAN Not. - POPL '14, vol. 49, no. 1, pp. 113-126, 2014.

[3] P. Quinn and U. Elzur, "Network Service Headers", https://www.ietf.org/id/draft-ietf-sfc-nsh-07.txt, Active Internet draft, 2016.

[4] "LINC-OE." [Online]. Available: https://www.opennetworking.org/sdnopenflow-products/778-infoblox-flowforwarding-linc. 